\title{
Handwritten Chinese Character Recognition: Effects of Shape Normalization and Feature Extraction
}

\author{
Cheng-Lin Liu \\ National Laboratory of Pattern Recognition (NLPR) \\ Institute of Automation, Chinese Academy of Sciences (CASIA) \\ PO Box 2728, Beijing 100080, P.R. China \\ E-mail: liucl@nlpr.ia.ac.cn
}

\begin{abstract}
The field of handwritten Chinese character recognition (HCCR) has seen significant advances in the last two decades, owing to the effectiveness of many techniques, especially those for character shape normalization and feature extraction. This paper reviews the major methods of normalization and feature extraction, and evaluates their performance experimentally. The normalization methods include linear normalization, nonlinear normalization (NLN) based on line density equalization, moment normalization (MN), bi-moment normalization $(\mathrm{BMN})$, modified centroid-boundary alignment (MCBA), and their pseudo-two-dimensional (pseudo 2D) extensions. As to feature extraction, we focus on some effective variations of direction features: chaincode feature, normalization-cooperated chaincode feature (NCCF), and gradient feature. We have compared the normalization methods previously, but in this study, will compare them with better implementation of features. As results, the current methods perform superiorly on handprinted characters, but are insufficient for unconstrained handwriting.
\end{abstract}

\section{Introduction}

Since the first work of printed Chinese character recognition (PCCR) was published in 1966 [1], many research efforts have been contributed, to both printed and handwritten Chinese character recognition (HCCR). Research on online HCCR was started as early as PCCR [2], whereas offline HCCR was started in late 1970s, and has attracted high attention from the 1980s [3]. Since then, many effective methods have been proposed to solve this problem, and the recognition performance has advanced significantly $[4,5]$. This paper is mainly concerned with offline HCCR, but most methods of offline recognition are applicable to online recognition as well [6].

The approaches of HCCR can be roughly grouped into two categories: feature matching (statistical classification) and structure analysis. Based on feature vector representation of character patterns, feature matching approaches usually computed a simple distance measure (correlation matching), say, Euclidean or city block distance, between the test pattern and class prototypes. Currently, sophisticated classification techniques $[7,8,9]$, including parametric and non-parametric statistical classifiers, neural networks, support vector machines (SVMs), etc., can yield higher recognition accuracies. Nevertheless, the selection and extraction of features remains an important issue. Structure analysis is an inverse process of character generation: to extract the constituent strokes and compute a structural distance measure between the test pattern and class models. Due to its resembling of human cognition and the potential of absorbing large deformation, this approach was pursued intensively in the 1980s and is still advancing [10]. However, due to the difficulty of stroke extraction and structural model building, it is not widely followed.

Statistical approaches have achieved great success in handprinted character recognition and are well commercialized due to some factors. First, feature extraction based on template matching and classification based on vector computation are easy to implement and computationally efficient. Second, effective shape normalization and feature extraction techniques, which improve the separability of patterns of different classes in feature space, have been proposed. Third, current machine learning methods enable classifier training with large set of samples for better discriminating shapes of different classes.

The methodology of Chinese character recognition has been largely affected by some important techniques: blurring [11], directional pattern match- 
ing $[12,13,14]$, nonlinear normalization $[15,16]$, modified quadratic discriminant function (MQDF) [17], etc. These techniques and their variations or improved versions, are still widely followed and adopted in most recognition systems. Blurring is actually a low-pass spatial filtering operation. It was proposed in the 1960s from the viewpoint of human vision, and is effective to blur the stroke displacement of characters of same class. Directional pattern matching, motivated from local receptive fields in vision, is the predecessor of current direction histogram features. Nonlinear normalization, which regulates stroke positions as well as image size, significantly outperforms the conventional linear normalization (resizing only). The MQDF is a nonlinear classifier suitable for high-dimensional features and large number of classes. Its variations include the pseudo Bayes classifier [18], the modified Mahalanobis distance [19], etc.

This paper reviews the major normalization and feature extraction methods and evaluate their performance in offline HCCR on large databases. The normalization methods include linear normalization (LN), nonlinear normalization (NLN) based on line density equalization $[15,16]$, moment normalization (MN) [20], bi-moment normalization (BMN) [21], modified centroid-boundary alignment (MCBA) [22], as well as the pseudo-two-dimensional (pseudo 2D) extensions of them [23, 24]. These methods have been evaluated previously [24], but in this study, they will be evaluated with better implementation of features.

Though many features have been proposed for character recognition, we focus on the class of direction histogram features, including chaincode direction feature, normalization-cooperated chaincode feature (NCCF) [25], and gradient direction feature. These features have yielded superior performance due to the sensitivity to stroke-direction variance and the insensitivity to stroke-width variance. The gradient direction feature was not paid enough attention until the success of gradient vector decomposition [26], following a decomposition scheme previously proposed in online character recognition [27]. Alternatively, the direction of gradient was quantized into a number of angular regions [28]. By NCCF, the chaincode direction is taken from the original image instead of the normalized image, but the directional elements are displaced in normalized planes according to normalized coordinates. An improved version of NCCF maps chaincodes into continuous line segments in normalized planes [29].

In the history, some extensions of direction feature, like the peripheral direction contributivity
(PDC) [30] and the reciprocal feature field [31], have reported higher accuracy in HCCR when simple distance metric was used. These features, with very high dimensionality (over 1,000), are actually highly redundant. As background features, they are sensitive to noise and connecting strokes. Extending the line element of direction feature to higher-order feature detectors (e.g., $[32,33]$ ) helps discriminate similar characters, but the dimensionality also increases rapidly. The Gabor filter, also motivated from vision research, promises feature extraction in character recognition [34], but is computationally expensive compared to chaincode and gradient features, and at best, perform comparably with the gradient feature [35].

We evaluate the character shape normalization and direction feature extraction methods on two databases of handwritten characters, ETL9B (Electrotechnical Laboratory, Japan) and CASIA (Institute of Automation, Chinese Academy of Sciences), with 3,036 classes and 3,755 classes, respectively. Two common classifiers, minimum distance classifier and modified quadratic discriminant function (MQDF), are used to evaluate the recognition accuracies.

The purpose of this study is twofold. First, the comparison of major normalization and feature extraction methods can provide guidelines for selecting methods in system development. Second, the results show to what degree of performance the state-ofthe-art methods can achieve. We will show in experiments that the current methods can recognize handprinted characters accurately but perform inferiorly on unconstrained handwriting.

In the rest of this paper, we review major normalization methods in Section 2 and direction feature extraction methods in Section 3. Experimental results are presented in Section 4, and finally, concluding remarks are offered in Section 5.

\section{Shape Normalization}

Normalization is to regulate the size, position, and shape of character images, so as to reduce the shape variation between the images of same class. Denote the input image and the normalized image by $f(x, y)$ and $g\left(x^{\prime}, y^{\prime}\right)$, respectively, normalization is implemented by coordinate mapping

$$
\left\{\begin{array}{l}
x^{\prime}=x^{\prime}(x, y) \\
y^{\prime}=y^{\prime}(x, y)
\end{array}\right.
$$


Most normalization methods use 1D coordinate mapping:

$$
\left\{\begin{array}{l}
x^{\prime}=x^{\prime}(x), \\
y^{\prime}=y^{\prime}(x) .
\end{array}\right.
$$

Under 1D normalization, the pixels at the same row/column in the input image are mapped to the same row/column in the normalized image, and hence, the shape restoration capability is limited.

Given coordinate mapping functions (1) or (2), the normalized image $g\left(x^{\prime}, y^{\prime}\right)$ is generated by pixel value and coordinate interpolation. In our implementation of $1 \mathrm{D}$ normalization, we map the coordinates forwardly from (binary) input image to normalized image, and use coordinate interpolation to generate the binary normalized image. For generating gray-scale normalized image, each pixel is viewed as a square of unit area. By coordinate mapping, the unit square of input image is mapped to a rectangle in the normalized plane, and each pixel (unit square) overlapping with the mapped rectangle is assigned a gray level proportional to the overlapping area [29].

In the case of 2D normalization, the mapped shape of a unit square onto the normalized plane is a quadrilateral [24]. To compute the overlapping areas of this quadrilateral with the pixels (unit squares) in the normalized plane, the quadrilateral is decomposed into trapezoids such that each trapezoid is within a row of unit squares. Each within-row trapezoid is further decomposed into trapezoids within a unit square. After generating the normalized grayscale image, the binary normalized image is obtained by thresholding the gray-scale image (fixed threshold 0.5 )

In our experiments, the normalized image plane is set to a square of edge length $L$, which is not necessarily fully occupied. To alleviate the distortion of elongated characters, we partially preserve the aspect ratio of the input image. By aspect ratio adaptive normalization (ARAN) [29, 36], the aspect ratio $R_{2}$ of normalized image is a continuous function of the aspect ratio $R_{1}$ of input image:

$$
R_{2}=\sqrt{\sin \left(\frac{\pi}{2} R_{1}\right)} .
$$

$R_{1}$ is calculated by

$$
R_{1}= \begin{cases}W_{1} / H_{1}, & \text { if } W_{1}<H_{1} \\ H_{1} / W_{1}, & \text { otherwise }\end{cases}
$$

where $W_{1}$ and $H_{1}$ are the width and height of the input image. The width $W_{2}$ and height $H_{2}$ of the normalized image are similarly related by the aspect ratio $R_{2}$. If the input image is vertically elongated, then in the normalized plane, the vertical dimension is filled (height $L$ ) and the horizontal dimension is centered and scaled according to the aspect ratio; otherwise, the horizontal dimension is filled (width $L)$ and the vertical dimension is centered and scaled. ARAN is depicted in Fig. 1.

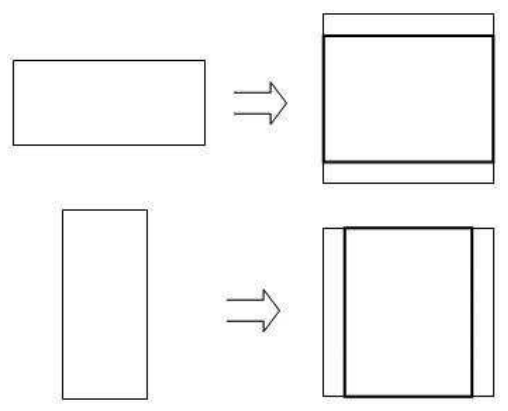

Figure 1: Aspect ratio adaptive normalization (ARAN). Rectangle with thick line: occupied area of normalized image.

The normalization methods depend on the coordinate mapping functions, defined by the $1 \mathrm{D}$ and pseudo 2D normalization methods as follows.

\subsection{D normalization methods}

Given the sizes of input and normalized images, the coordinate mapping functions of linear normalization (LN) are simply

$$
\left\{\begin{array}{l}
x^{\prime}=\frac{W_{2}}{W_{1}} x \\
y^{\prime}=\frac{H_{2}}{H_{1}} y
\end{array}\right.
$$

Both the linear normalization and line-density-based nonlinear normalization (NLN) methods align the physical boundaries (ends of stroke projections) of input image to the boundaries of normalized image. The coordinate mapping of NLN is obtained by accumulating the normalized line density projections (line density equalization):

$$
\left\{\begin{array}{l}
x^{\prime}=W_{2} \sum_{u=0}^{x} h_{x}(u) \\
y^{\prime}=H_{2} \sum_{v=0}^{y} h_{y}(v)
\end{array}\right.
$$

where $h_{x}(x)$ and $h_{y}(y)$ are the normalized line density histograms of $x$ axis and $y$ axis, respectively, which are obtained by normalizing the projections of local line densities into unity sum:

$$
\left\{\begin{array}{l}
h_{x}(x)=\frac{p_{x}(x)}{\sum_{x} p_{x}(x)}=\frac{\sum_{y} d_{x}(x, y)}{\sum_{x} \sum_{y} d_{x}(x, y)}, \\
h_{y}(y)=\frac{p_{y}(y)}{\sum_{y} p_{y}(y)}=\frac{\sum_{x} d_{y}(x, y)}{\sum_{x} \sum_{y} d_{y}(x, y)},
\end{array}\right.
$$

where $p_{x}(x)$ and $p_{y}(y)$ are the line density projections onto $x$ axis and $y$ axis, respectively, and $d_{x}(x, y)$ and $d_{y}(x, y)$ are local line density functions. 
By Tsukumo and Tanaka [15], the local line densities $d_{x}$ and $d_{y}$ are taken as the reciprocal of horizontal/vertical run-length in background area, or a small constant in stroke area. While by Yamada et al. [16], $d_{x}$ and $d_{y}$ are calculated considering both background run-length and stroke run-length, and are unified to render $d_{x}(x, y)=d_{y}(x, y)$. The two methods perform comparably but the one of Tsukumo and Tanaka is computationally simpler [5]. By adjusting the density functions of marginal and stroke areas empirically in Tsukumo and Tanaka's method, we have achieved better performance than the method of Yamada et al. This improved version of NLN is taken in our experiments.

The 1D moment normalization (MN) method (a simplified version of Casey's method [20]) aligns the centroid of input image $\left(x_{c}, y_{c}\right)$ to the geometric center of normalized image $\left(x_{c}^{\prime}, y_{c}^{\prime}\right)=\left(W_{2} / 2, H_{2} / 2\right)$, and re-bound the input image according to secondorder 1D moments. Let the second-order moments be $\mu_{20}$ and $\mu_{02}$, the width and height of input image are re-set to $\delta_{x}=4 \sqrt{\mu_{20}}$ and $\delta_{y}=4 \sqrt{\mu_{02}}$, respectively. The coordinate mapping functions are then given by

$$
\left\{\begin{aligned}
x^{\prime} & =\frac{W_{2}}{\delta_{x}}\left(x-x_{c}\right)+x_{c}^{\prime}, \\
y^{\prime} & =\frac{H_{2}}{\delta_{y}}\left(y-y_{c}\right)+y_{c}^{\prime} .
\end{aligned}\right.
$$

The bi-moment normalization (BMN) method [21] aligns the centroid of input image as moment normalization does, but the width and height are treated asymmetric with respect to the centroid. To do this, the second-order moments are split into two parts by the centroid: $\mu_{x}^{-}, \mu_{x}^{+}, \mu_{y}^{-}$, and $\mu_{y}^{+}$. The boundaries of input image are re-set to $\left[x_{c}-\right.$ $\left.2 \sqrt{\mu_{x}^{-}}, x_{c}+2 \sqrt{\mu_{x}^{+}}\right]$and $\left[y_{c}-2 \sqrt{\mu_{y}^{-}}, y_{c}+2 \sqrt{\mu_{y}^{+}}\right]$. For the $x$ axis, a quadratic function $u(x)=a x^{2}+b x+c$ is used to align three points $\left(x_{c}-2 \sqrt{\mu_{x}^{-}}, x_{c}, x_{c}+\right.$ $\left.2 \sqrt{\mu_{x}^{+}}\right)$to normalized coordinates $(0,0.5,1)$, and similarly, a quadratic function $v(y)$ is used for the $y$ axis. Finally, the coordinate functions are

$$
\left\{\begin{array}{l}
x^{\prime}=W_{2} u(x), \\
y^{\prime}=H_{2} v(y) .
\end{array}\right.
$$

The quadratic functions can also be used to align the physical boundaries and centroid, i.e., map $\left(0, x_{c}, W_{1}\right)$ and $\left(0, y_{c}, H_{1}\right)$ to $(0,0.5,1)$. We call this method centroid-boundary alignment (CBA). A modified CBA (MCBA) method [22] also adjusts the stroke density in central area by combining a sine function with the quadratic functions:

$$
\left\{\begin{array}{l}
x^{\prime}=W_{2}\left[u(x)+\eta_{x} \sin (2 \pi u(x))\right], \\
y^{\prime}=H_{2}\left[v(y)+\eta_{y} \sin (2 \pi v(y))\right] .
\end{array}\right.
$$

The amplitudes of sine waves, $\eta_{x}$ and $\eta_{y}$, are estimated from the extent of the central area, which is defined by the centroids of partial images divided by the global centroid.

\subsection{Pseudo 2D normalization meth- ods}

Horiuchi et al. proposed a pseudo 2D nonlinear normalization (P2DNLN) method by equalizing the line density functions of each row/column instead of the line density projections [23]. To control the degree of shape deformation, they blurred the line density functions such that the equalization of each row/column is dependent on its neighboring rows/columns. Though this method promises recognition, it is computationally expensive due to the row/column-wise line density blurring.

An efficient pseudo 2D normalization approach, called line density projection interpolation (LDPI), was proposed recently [24]. Instead of line density blurring and row/column-wise equalization, LDPI partitions the 2D line density map into soft strips. 1D coordinate functions are computed from the density projection of each strip and combined to a $2 \mathrm{D}$ function. Specifically, let the width and height of the input image be $W_{1}$ and $H_{1}$, the centroid be $\left(x_{c}, y_{c}\right)$, we partition the horizontal line density map $d_{x}(x, y)$ into three horizontal strips:

$$
d_{x}^{i}(x, y)=w^{i}(y) d_{x}(x, y), \quad i=1,2,3 .
$$

$w^{i}(y)(i=1,2,3)$ are piecewise linear functions:

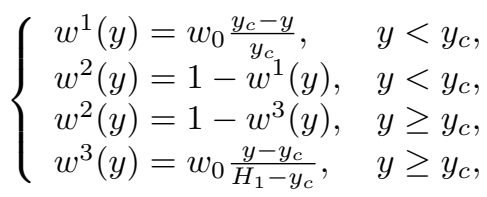

where $w_{0}$ controls the weight of the upper/lower part of line density map. A small value of $w_{0}$ renders the interpolated 2D coordinate function close to that of 1D normalization, while a large one may yield excessive deformation. The weight functions with $w_{0}=1$ are depicted in Fig. 2 .

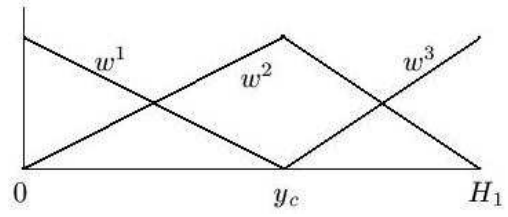

Figure 2: Weight functions for partitioning line density map into soft strips.

The horizontal density functions of three strips are 
projected onto the $x$ axis:

$$
p_{x}^{i}(x)=\sum_{y} d_{x}^{i}(x, y), \quad i=1,2,3 .
$$

The projections are then normalized to unity sum and accumulated to give $1 \mathrm{D}$ coordinate functions $x^{\prime i}(x), i=1,2,3$, which are combined to $2 \mathrm{D}$ coordinate function by interpolation:

$$
x^{\prime}(x, y)= \begin{cases}w^{1}(y) x^{\prime 1}(x)+w^{2}(y) x^{\prime 2}(x), & y<y_{c} \\ w^{3}(y) x^{\prime 3}(x)+w^{2}(y) x^{\prime 2}(x), & y \geq y_{c} .\end{cases}
$$

Similar to the partitioning of horizontal density, the vertical density map $d_{y}(x, y)$ is partitioned into three vertical strips using weight functions in $x$ axis. The partitioned density functions $d_{y}^{i}(x, y)$, $i=1,2,3$, are similarly equalized and interpolated to generate the $2 \mathrm{D}$ coordinate function $y^{\prime}(x, y)$.

The strategy of LDPI is applied to extend other 1D normalization methods: MN, BMN, and MCBA. The extended versions are called pseudo 2D MN (P2DMN), pseudo 2D BMN (P2DBMN), and pseudo 2D CBA (P2DCBA), respectively. These methods do not rely on the computation of local line density map. Instead, they are directly based on the pixel intensity of character image. As the soft partitioning of line density map in LDPI, the input character image $f(x, y)$ is softly partitioned into three horizontal strips $f_{x}^{i}(x, y), i=1,2,3$, and three vertical strips $f_{y}^{i}(x, y), i=1,2,3$. The horizontal strips are projected onto the $x$ axis:

$$
p_{x}^{i}(x)=\sum_{y} f_{x}^{i}(x, y), \quad i=1,2,3 .
$$

For P2DMN, the second order moment is computed from the projection of a strip:

$$
\mu_{20}^{i}=\frac{\sum_{x}\left(x-x_{c}^{i}\right)^{2} p_{x}^{i}(x)}{\sum_{x} p_{x}^{i}(x)} .
$$

The width of this strip is re-set to $\delta_{x}^{i}=4 \sqrt{\mu_{20}^{i}}$, which is used to determine the scaling factor of $1 \mathrm{D}$ coordinate mapping:

$$
x^{i}(x)=\frac{W_{2}}{\delta_{x}^{i}}\left(x-x_{c}^{i}\right)+\frac{W_{2}}{2} .
$$

And the 1D coordinate functions of vertical strips are computed from strip projections similarly.

For P2DBMN, the second order moment of a horizontal strip is split into two parts at the centroid of this strip: $\mu_{20}^{i-}$ and $\mu_{20}^{i+}$. The bounds of this strip is re-set to $\left[x_{c}^{i}-2 \sqrt{\mu_{20}^{i-}}, x_{c}^{i}+2 \sqrt{\mu_{20}^{i+}}\right]$, which, together with the centroid $x_{c}^{i}$, are used to estimate the quadratic $1 \mathrm{D}$ coordinate mapping function $x^{\prime i}(x)$.
The three 1D coordinate functions of vertical strips are computed similarly.

By P2DCBA, from the vertical projection of each horizontal strip $f_{x}^{i}(x, y)(i=1,2,3)$, the centroid coordinate $x_{c}^{i}$ and two partial centroids $x_{c 1}^{i}$ and $x_{c 2}^{i}$ are computed to estimate the parameters of $1 \mathrm{D}$ coordinate mapping function $x^{\prime i}(x)$. Similarly, 1D coordinate functions $y^{\prime i}(y)(i=1,2,3)$ are estimated from the horizontal projections of vertical strips.

More details of pseudo 2D normalization can be found in [24]. Some examples of normalization using nine methods (LN, NLN, MN, BMN, MCBA, LDPI, P2DMN, P2DBMN, and P2DCBA) are shown in Fig. 3. We can see that whereas linear normalization (LN) keeps the original shape (only aspect ratio changed), NLN can effectively equalize the line intervals. The centroid-based normalization methods (MN, BMN, and MCBA) effectively regulate the overall shape (skewness of gravity, balance of inner/outer stroke density). The pseudo 2D methods make the stroke positions more uniform, especially, alleviate the imbalance of width/height and position of character parts.

\section{Direction Feature Extrac- tion}

The implementation of direction feature is varying depending on the directional element decomposition, the sampling of feature values, the resolution of direction and feature plane, etc. Considering that the stroke segments of Chinese characters can be approximated into four orientations: horizontal, vertical, left-diagonal and right-diagonal, early works usually decomposed the stroke (or contour) segments into four orientations.

Feature extraction from stroke contour has been widely adopted because the contour length is nearly independent on stroke-width variation. The local direction of contour, encoded as a chaincode, actually has eight directions (Fig. 4). Decomposing the contour pixels into eight directions instead of four orientations (a pair of opposite directions merged into one orientation) was shown to significantly improve the recognition accuracy [26]. This is because separating the two sides of stroke edge can better discriminate parallel strokes. The direction of stroke edge can also be measured by the gradient of image intensity, which applies to gray-scale image as well binary image. The gradient feature has been applied to Chinese character recognition in 8-direction [37] and 12-direction [38].

Direction feature extraction is accomplished in 


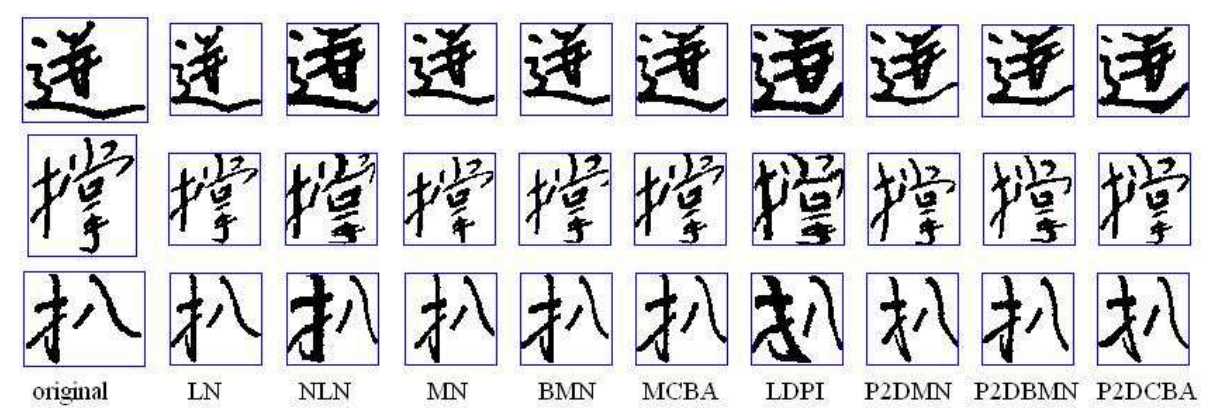

Figure 3: Character image normalization by nine methods. The leftmost image is original and the other eight are normalized ones.

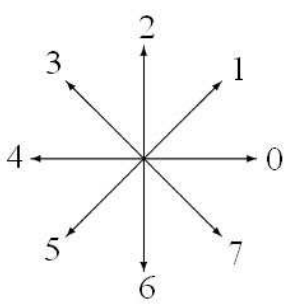

Figure 4: Eight directions of chaincodes.

three steps: image normalization, directional decomposition, and feature sampling. Conventionally, the contour/edge pixels of normalized image are assigned to a number of direction planes. The normalization-cooperated feature extraction (NCFE) strategy [25], instead, assigns the chaincodes of original image into direction planes. Though the normalized image is not generated by NCFE, the coordinates of pixels in original image are mapped to a standard plane, and the extracted feature is thus dependent on the normalization method.

Direction feature is also called direction histogram feature because at a pixel or a local region in normalized image, the strength values of $N_{d}$ directions form a local histogram. Alternatively, we view the strength values of one direction as a directional image (direction plane).

In the following, we first describe the directional decomposition procedures for three types of direction features: chaicode direction feature, normalization-cooperated chaincode feature $(\mathrm{NCCF})$, and gradient direction feature, and then address the sampling of direction planes.

\subsection{Directional decomposition}

Directional decomposition results in a number of direction planes (with same size as the normalize image), $f_{i}(x, y), i=1, \ldots, N_{d}$. We first describe the procedures for decomposing contour/gradient into eight directions, then extend to 12 directions and 16 directions.

In binary image, a contour pixel is a black point with at least one of its 4-connected neighbors being white. The 8-direction chaincodes of contour pixels can be decided by contour tracing, or more simply, by raster scan [39]. At a black pixel $(x, y)$, denoting the values of 8-connected neighbors in counterclockwise as $p_{k}, 0,1, \ldots, 7$, with the east neighbor being $p_{0}$. For $k=0,2,4,6$, if $p_{k}=0$, check $p_{k+1}$ : if $p_{k+1}=1$ (chaincode $\left.k+1\right), f_{k+1}(x, y)$ increases by 1 ; otherwise, if $p_{(k+2) \% 8}=1$ (chaincode $(k+2) \% 8$ ), $f_{(k+2) \% 8}(x, y)$ increases by 1 .

For NCCF, each chaincode in the original image is viewed as a line segment connecting two neighboring pixels, which is mapped to another line segment in a standard direction plane by coordinate mapping. In the direction plane, each pixel (unit square) crossed by the line segment in the main ( $x$ or $y$ ) direction is given a unit of direction contribution. To exploit the continuous nature of line segment, the strength of line direction falling in a pixel is proportional to the length of line segment falling in the unit square (continuous NCCF [29]). As in Fig. 5, where a line segment mapped from a chaincode covers four unit squares A, B, C and D. By discrete NCCF, the pixels $\mathrm{A}$ and $\mathrm{C}$ are assigned a direction unit, whereas by continuous NCCF, all the four pixels are assigned direction strengths proportional to the in-square line segment length.

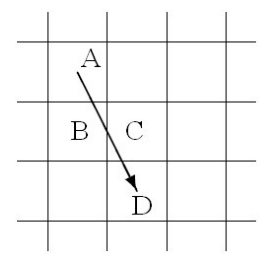

Figure 5: NCCF on continuous direction plane.

In gradient direction feature extraction, the gradi- 
ent vector, computed on the normalized image using the Sobel operator, is decomposed into components in eight chaincode directions. The Sobel operator has two masks to compute the gradient components in two axes. The masks are shown in Fig. 6, and the gradient $\mathbf{g}(x, y)=\left[g_{x}, g_{y}\right]^{T}$ at location $(x, y)$ is computed by

$$
\begin{aligned}
& g_{x}(x, y) \\
& =f(x+1, y-1)+2 f(x+1, y)+f(x+1, y+1) \\
& -f(x-1, y-1)-2 f(x-1, y)+f(x-1, y+1) \\
& g_{y}(x, y) \\
& =f(x-1, y+1)+2 f(x, y+1)+f(x+1, y+1) \\
& -f(x-1, y-1)-2 f(x, y-1)+f(x+1, y-1)
\end{aligned}
$$

\begin{tabular}{|r|r|r|}
\hline-1 & 0 & 1 \\
\hline-2 & 0 & 2 \\
\hline-1 & 0 & 1 \\
\hline
\end{tabular}

\begin{tabular}{|r|r|r|}
\hline 1 & 2 & 1 \\
\hline 0 & 0 & 0 \\
\hline-1 & -2 & -1 \\
\hline
\end{tabular}

Figure 6: Templates of Sobel gradient operator.

The gradient strength and direction can be computed from the vector $\left[g_{x}, g_{y}\right]^{T}$. The range of gradient direction can be partitioned into a number (say, 8 or 16) of regions and each region corresponds to a direction plane. More effectively, the gradient vector is decomposed into components in standard directions, following a strategy previously proposed in online character recognition [27]. In this scheme, if a gradient direction lies between two standard directions, the vector is decomposed into two components as shown in Fig. 7. The length of each component is assigned to the corresponding direction plane at the pixel $(x, y)$.

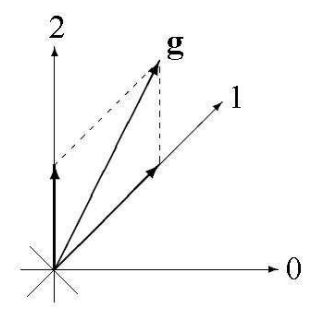

Figure 7: Decomposition of gradient vector.

Fig. 8 shows the direction planes of three decomposition schemes: NCCF, chaincodes of normalization image, and gradient of normalized image. The direction planes are arranged in the order of stroke orientation. We can see that the planes of chaincode directions (third row) are very similar to those of gradient directions. The planes of NCCF, describing the local directions of original image, show some difference. Comparing the original image and the normalized image, the orientation of the righthand stroke, near left-diagonal orientation, deforms to near vertical. Consequently, the direction planes of left-diagonal orientation of NCCF are stronger than those of chaincodes and gradient, while the planes of vertical orientation of NCCF are weaker than those of chaincodes and gradient.

\subsection{Extention to more directions}

The extension of gradient decomposition into more than eight directions is straightforward: simply setting $N_{d}$ standard directions with angle interval $360 / N_{d}$ and typically, with one direction pointing to east, then decompose each gradient vector into two components in standard directions and assign the component lengths to corresponding direction planes. We set $N_{d}$ to 12 and 16 .

To decompose contour pixels into 16 directions follows the 16-direction extended chaincodes, which is defined by two consecutive chaincodes. In the weighted direction histogram feature of Kimura et al. [18], 16-direction chaincodes are down-sampled by weighted average to form 8-direction planes.

Again, we can determine the 16-direction chaincode of contour pixels by raster scan. At a contour pixel $(x, y)$, when its 4-connected neighbor $p_{k}=0$ and the counterclockwise successor $p_{k+1}=1$ or $p_{(k+2) \% 2}=1$, search the neighbors clockwise from $p_{k}$ until a $p_{j}=1$ is found. The two contour pixels, $p_{k+1}$ or $p_{(k+2) \% 2}$ and $p_{j}$, form a 16 -direction chaincode. For example, in Fig. 9, the center pixel has the east neighbor being 0 , the north neighbor alone defining the 8-direction chaincode, and defining a 16-direction chaincode together with the southeast neighbor. The 16-direction chaincode can be indexed from a table of correspondence between the code and the difference of coordinates of two pixels forming the code, as shown in Fig. 10. Each contour pixel has a unique 16-direction code.

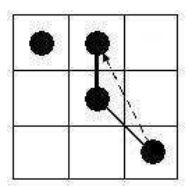

Figure 9: 16-direction chaincode formed from two 8-direction chaincodes.

For decomposing contour pixels into 12 directions, the difference of coordinates corresponding to a 16direction chaincode is viewed as a vector (the dashed line in Fig. 9), which is decomposed into components in 12 standard directions as a gradient vector 


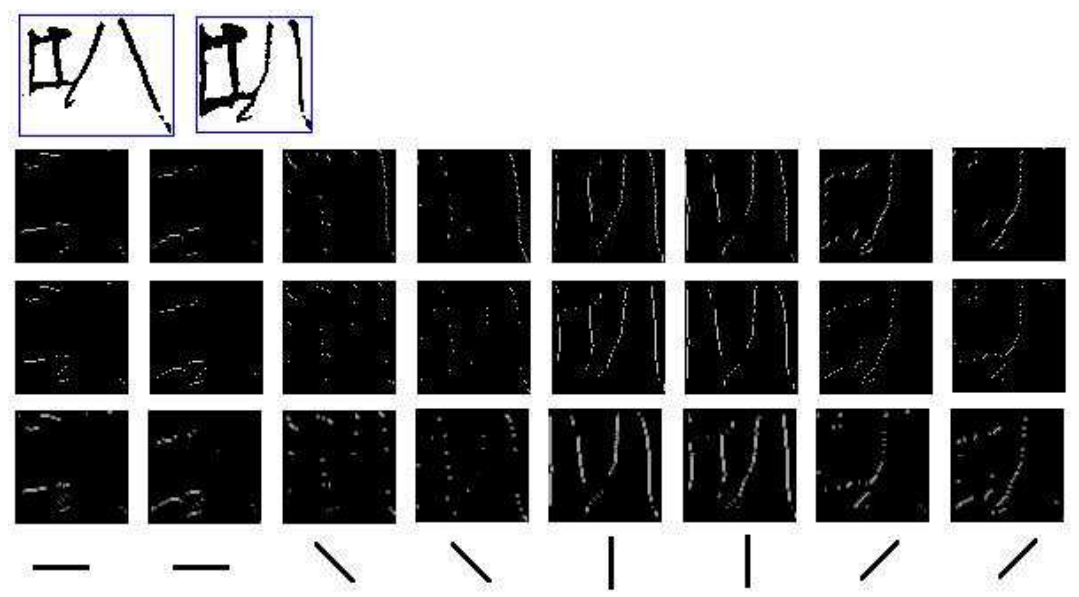

Figure 8: Original image and normalized image (top row), 8-direction planes of NCCF (second row), chaincode planes of normalized image (third row), and gradient direction planes (bottom row).

\begin{tabular}{|c|c|c|c|c|}
\hline $\begin{array}{c}(-2,2) \\
6\end{array}$ & $\begin{array}{c}(-1,2) \\
5\end{array}$ & $\begin{array}{c}0,2) \\
4\end{array}$ & $\begin{array}{c}1,2) \\
3\end{array}$ & $\begin{array}{c}(2,2) \\
2\end{array}$ \\
\hline$(-2,1)$ & $(-1,1)$ & $(0,1)$ & $(1,1)$ & $(2,1)$ \\
7 & 6 & 4 & 2 & 1 \\
\hline $\begin{array}{c}(-2,0) \\
8\end{array}$ & $(-1,0)$ & $(0,0)$ & $(1,0)$ & $(2,0)$ \\
\hline$(-2,-1)$ & $(-1,-1)$ & $(0,-1)$ & $(1,-1)$ & $(2,-1)$ \\
9 & 10 & 12 & 14 & 15 \\
\hline$(-2,-2)$ & $(-1,-2)$ & $(0,-2)$ & $(1,-2)$ & $(2,-2)$ \\
10 & 11 & 12 & 13 & 14 \\
\hline
\end{tabular}

Figure 10: Difference of coordinates and the corresponding 16-direction chaincodes.

is done. In this sense, the 12-direction code of a contour pixel is not unique. For 12-direction chaincode feature extraction, a contour pixel is assigned to two direction planes, with strength proportional to the component length. For NCCF, the two corresponding direction planes are assigned strengths proportional to the overlapping length of the line segment mapped by coordinate functions, as in Fig. 5 .

\subsection{Blurring and sampling}

Each direction plane, with same size as the normalized image, need to be reduced to extract feature values of moderate dimensionality. A simple way is to partition the direction plane into a number of block zones and take the total or average value of each zone as a feature value. Partition of variable-size zones was proposed to overcome the non-uniform distribution of stroke density [13], but is not necessary when nonlinear or pseudo 2D normalization is done. Overlapping blocks were taken to alleviate the effect of stroke-position variation on the boundary of blocks [40], yet a more effective way is to partition the plane into soft zones, which follow the principle of low-pass spatial filtering and sampling [39]. The blurring operation of Iijima [11] implies spatial filtering without down-sampling.

In implementation of blurring, the impulse response function (IRF) of spatial filter is approximated into a weighted window, which is also called a blurring mask. The IRF is often taken as a Gaussian function:

$$
h(x, y)=\frac{1}{2 \pi \sigma_{x}^{2}} \exp \left(-\frac{x^{2}+y^{2}}{2 \sigma_{x}^{2}}\right) .
$$

According to the Sampling Theorem, the variance parameter $\sigma_{x}$ is related to the sampling frequency (the reciprocal of sampling interval). On truncating the band-width of Gaussian filter, an empirical formula was given in [39]:

$$
\sigma_{x}=\frac{\sqrt{2} t_{x}}{\pi}
$$

where $t_{x}$ is the sampling interval. At a location $\left(x_{0}, y_{0}\right)$ of image $f(x, y)$, the convolution gives a sampled feature value

$$
F\left(x_{0}, y_{0}\right)=\sum_{x} \sum_{y} f(x, y) \cdot h\left(x-x_{0}, y-y_{0}\right)
$$

Fig. 11 show the blurred images (without downsampling) of the direction planes in Fig. 8. By blurring, the sparse pixels in direction planes merge into strokes or blobs.

For ease of implementation, we partition a direction plane into a mesh of equal-size blocks and set the sampling points to the center of each block. Assume to extract $K \times K$ values from a plane, the size of plane is set to $K t_{x} \times K t_{x}$. From $N_{d}$ direction 

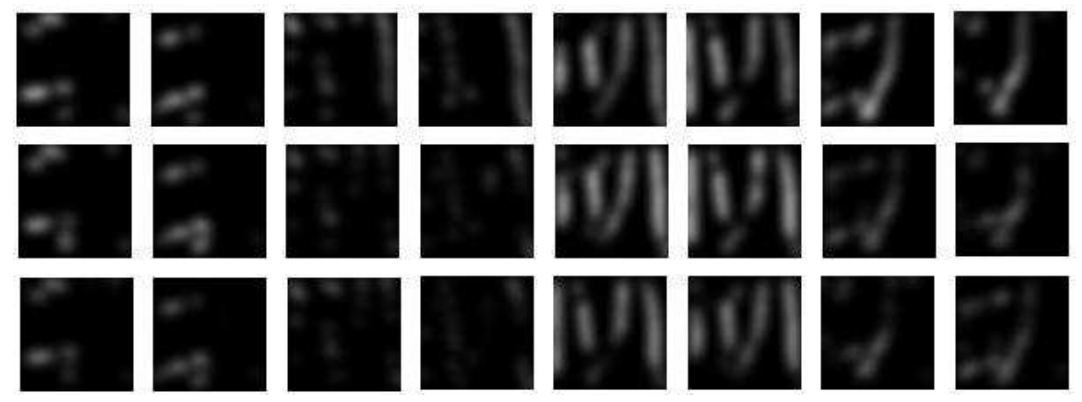

Figure 11: Blurred images (not down-sampled) of the direction planes in Fig. 8.

planes, the total number of extracted feature values is $N_{d} \times K^{2}$.

The extracted feature values are causal variables. Power transformation can make the density function of causal variables closer to Gaussian [7]. This is helpful to improve the classification performance of statistical classifiers. Power transformation is also called variable transformation [41] or Box-Cox transformation [42]. We transform each feature value with power 0.5 without attempt to optimize the transformation functions.

\section{Performance Evaluation}

We first compare the performance of the normalization and feature extraction methods on two large databases of handprinted characters (constrained writing), and then test the performance on a small set of unconstrained handwritten characters.

We use two classifiers for classification: the Euclidean distance to class mean (minimum distance classifier) and the MQDF [17]. For reducing the classifier complexity and improving classification accuracy, the feature vector is transformed to a lower dimensionality by Fisher linear discriminant analysis (FLDA) [7]. We set the reduced dimensionality to 160 for all feature types.

Denote the $d$-dimensional feature vector (after dimensionality reduction) by $\mathbf{x}$, the MQDF of class $\omega_{i}$ is computed by

$$
\begin{aligned}
g_{2}\left(\mathbf{x}, \omega_{i}\right) & =\sum_{j=1}^{k} \frac{1}{\lambda_{i j}}\left[\left(\mathbf{x}-\mu_{i}\right)^{T} \phi_{i j}\right]^{2} \\
& +\frac{1}{\delta_{i}}\left\{\left\|x-\mu_{i}\right\|^{2}-\sum_{j=1}^{k}\left[\left(\mathbf{x}-\mu_{i}\right)^{T} \phi_{i j}\right]^{2}\right\} \\
& +\sum_{j=1}^{k} \log \lambda_{i j}+(d-k) \log \delta_{i},
\end{aligned}
$$

where $\mu_{i}$ is the mean vector of class $\omega_{i}, \lambda_{i j}$ and $\phi_{i j}, j=1, \ldots, d$, are the eigenvalues and eigenvectors of the covariance matrix of class $\omega_{i}$. The eigenvalues are sorted in non-ascending order and the eigenvectors are sorted accordingly. $k$ denotes the number of principal axes, and the minor eigenvalues are replaced with a constant $\delta_{i}$. We set a class-independent constant $\delta_{i}$ which is proportional to the average feature variance, with the multiplier selected by 5 -fold holdout validation on the training data set.

In our experiments, $k$ was set to 40 . The classification of MQDF is speeded up by selecting 100 candidate classes using Euclidean distance. The MQDF is then computed on the candidate classes only. Candidate selection is further accelerated by clustering the class means into groups. The input feature vector is first compared to cluster centers, and then compared to the class means contained in a number of nearest clusters. We set the total number of clusters to 220 for the ETL9B database and 250 for the CASIA database.

MQDF is a promising classification method for HCCR. Even higher performance can be achieved by, e.g., discriminative learning of feature transformation and classifier parameters [37, 38]. The optimization of classifier, however, is not the concern of this paper.

\subsection{Performance on handprinted characters}

The normalization and feature extraction methods are evaluated on two databases of handprinted characters. The ETL9B database contains the character images of 3,036 classes (71 hiragana, and 2,965 Kanji characters in the JIS level-1 set), 200 samples per class. This database has been widely evaluated by the community [18, 40, 43]. The CASIA database, collected by the Institute of Automation, Chinese Academy of Sciences, in early 1990s, contains the handwritten images of 3,755 Chinese characters (the level-1 set in GB2312-80 standard), 300 samples per class. Some sample images of the CASIA database are shown in Fig. 12.

In the ETL9B database, we use the first 20 and last 20 samples of each class for testing, and the re- 


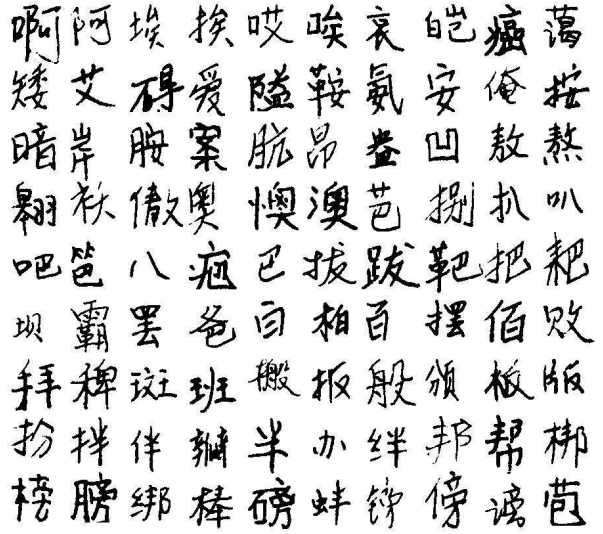

Figure 12: Test samples in the CASIA database.
Table 2: Error rates (\%) of 8-direction, 12-direction, and 16-direction features on ETL9B database.

\begin{tabular}{|c|ccc|ccc|}
\hline & \multicolumn{3}{|c|}{ Euclidean } & \multicolumn{3}{c|}{ MQDF } \\
\hline chn & M1 & M2 & M3 & M1 & M2 & M3 \\
\hline 8-dir & 2.91 & 2.94 & 3.02 & 1.08 & 1.05 & 1.09 \\
12-dir & 2.43 & 2.56 & 2.54 & 1.02 & 1.00 & $\mathbf{0 . 9 7}$ \\
16-dir & 2.52 & $\mathbf{2 . 4 0}$ & 2.52 & 1.20 & 1.00 & 1.00 \\
\hline nccf & M1 & M2 & M3 & M1 & M2 & M3 \\
\hline 8-dir & 2.61 & 2.61 & 2.71 & 0.93 & 0.87 & 0.89 \\
12-dir & 2.05 & 2.06 & 2.13 & 0.82 & $\mathbf{0 . 7 7}$ & 0.78 \\
16-dir & 2.05 & $\mathbf{2 . 0 4}$ & 2.11 & 0.98 & 0.85 & 0.79 \\
\hline grd-g & M1 & M2 & M3 & M1 & M2 & M3 \\
\hline 8-dir & 2.59 & 2.58 & 2.66 & 0.93 & 0.90 & 0.89 \\
12-dir & 2.27 & 2.30 & 2.31 & 0.94 & 0.86 & 0.86 \\
16-dir & 2.29 & $\mathbf{2 . 1 9}$ & 2.25 & 1.08 & 0.94 & $\mathbf{0 . 8 5}$ \\
\hline
\end{tabular}

maining samples for training classifiers. In the CASIA database, we use the first 250 samples of each class for training, and the remaining 50 samples per class for testing.

We first compare the performance of three direction features with varying direction resolutions with a common normalization method (Tsukumo and Tanaka's nonlinear normalization (NLN) method with our modifications). The direction resolution of features is set to 8,12 , and 16 . For each direction resolution, three schemes of sampling mesh are tested. For 8-direction features, the mesh of sampling is set to $7 \times 7(\mathrm{M} 1), 8 \times 8(\mathrm{M} 2)$, and $9 \times 9(\mathrm{M} 3)$; for 12-direction, $6 \times 6(\mathrm{M} 1), 7 \times 7(\mathrm{M} 2)$, and $8 \times 8$ (M3); and for 16-direction, $5 \times 5$ (M1), $6 \times 6(\mathrm{M} 2)$, and $7 \times 7$ (M3). We control the size of normalized image (direction planes) to be around $64 \times 64$, and the dimensionality (before reduction) to be less than 800. The settings of sampling mesh are summarized in Table 1.

Table 1: Settings of sampling mesh for 8-direction, 12-direction, and 16-direction features.

\begin{tabular}{|c|cc|cc|cc|}
\hline \multirow{2}{*}{ Mesh } & \multicolumn{2}{|c|}{ M1 } & \multicolumn{2}{c|}{ M2 } & \multicolumn{2}{c|}{ M3 } \\
\cline { 2 - 7 } & zones & dim & zones & dim & zones & dim \\
\hline 8-dir & $7 \times 7$ & 392 & $8 \times 8$ & 512 & $9 \times 9$ & 648 \\
12-dir & $6 \times 6$ & 432 & $7 \times 7$ & 588 & $8 \times 8$ & 768 \\
16-dir & $5 \times 5$ & 400 & $6 \times 6$ & 576 & $7 \times 7$ & 784 \\
\hline
\end{tabular}

On classifier training and testing using different direction resolutions and sampling schemes, the error rates on the test set of ETL9B database are listed in Table 2, and the error rates on the test set of CASIA database are listed in Table 3. In the tables, the chaincode direction feature is denoted by chn, NCCF is denoted by nccf, and the gradient direc-
Table 3: Error rates (\%) of 8-direction, 12-direction, and 16-direction features on CASIA database.

\begin{tabular}{|c|ccc|ccc|}
\hline & \multicolumn{3}{|c|}{ Euclidean } & \multicolumn{3}{c|}{ MQDF } \\
\hline chn & M1 & M2 & M3 & M1 & M2 & M3 \\
\hline 8-dir & 5.95 & 6.07 & 6.22 & 2.45 & 2.37 & 2.37 \\
12-dir & 5.34 & 5.44 & 5.53 & 2.34 & 2.26 & $\mathbf{2 . 2 1}$ \\
16-dir & 5.44 & $\mathbf{5 . 2 8}$ & 5.37 & 2.72 & 2.35 & 2.29 \\
\hline nccf & M1 & M2 & M3 & M1 & M2 & M3 \\
\hline 8-dir & 5.31 & 5.35 & 5.49 & 1.94 & 1.92 & 2.00 \\
12-dir & 4.44 & 4.48 & 4.55 & 1.86 & 1.75 & $\mathbf{1 . 7 3}$ \\
16-dir & 4.53 & $\mathbf{4 . 4 2}$ & 4.52 & 2.17 & 1.92 & 1.82 \\
\hline grd-g & M1 & M2 & M3 & M1 & M2 & M3 \\
\hline 8-dir & 5.31 & 5.34 & 5.41 & 2.05 & 2.01 & 1.97 \\
12-dir & 4.94 & 4.90 & 4.98 & 2.09 & 2.00 & $\mathbf{1 . 9 5}$ \\
16-dir & 4.98 & 4.85 & $\mathbf{4 . 8 0}$ & 2.42 & 2.09 & 1.98 \\
\hline
\end{tabular}

tion feature by grd-g. For chaincode feature extraction, the normalized binary image is smoothed using a connectivity-preserving smoothing algorithm [39]. Gradient feature is extracted from gray-scale normalized image.

We can see that on either database, using either classifier (Euclidean or MQDF), the error rates of 12direction and 16-direction features are mostly lower than those of 8-direction features. This indicates that increasing the resolution of direction decomposition is beneficial. The 16-direction feature, however, does not outperform the 12-direction feature. To select a sampling mesh, let us focus on the results of 12-direction features. We can see that by Euclidean distance classification, M1 $(6 \times 6)$ outperforms M2 $(7 \times 7)$ and M3 $(8 \times 8)$, whereas by MQDF, the error rates of M2 and M3 are lower than those of M1. Considering that M2 and M3 perform comparably while M2 has lower complexity, we take the sampling mesh M2 with 12-direction features for fol- 
lowing experiments. The original dimensionality of direction features is now $12 \times 7 \times 7=588$.

Table 4: Error rates (\%) of various normalization methods on ETL9B database.

\begin{tabular}{|c|ccc|ccc|}
\hline & \multicolumn{3}{|c|}{ Euclidean } & \multicolumn{3}{c|}{ MQDF } \\
\cline { 2 - 7 } & chn & nccf & grd-g & chn & nccf & grd-g \\
\hline LN & 6.36 & 5.94 & 5.97 & 2.38 & 2.09 & 2.11 \\
NLN & 2.56 & 2.06 & 2.30 & 1.00 & 0.77 & 0.86 \\
MN & 2.35 & 2.07 & 2.12 & 0.95 & 0.83 & 0.82 \\
BMN & 2.33 & 2.04 & 2.09 & 0.92 & 0.81 & 0.80 \\
MCBA & 2.52 & 2.19 & 2.27 & 1.00 & 0.84 & 0.86 \\
\hline LDPI & 2.08 & 1.65 & 1.90 & $\mathbf{0 . 8 2}$ & $\mathbf{0 . 6 4}$ & $\mathbf{0 . 7 3}$ \\
2MN & 2.05 & 1.65 & 1.84 & 0.86 & 0.69 & 0.74 \\
2BMN & $\mathbf{1 . 9 7}$ & $\mathbf{1 . 6 0}$ & $\mathbf{1 . 7 8}$ & 0.84 & 0.69 & $\mathbf{0 . 7 3}$ \\
2CBA & 2.13 & 1.81 & 1.93 & 0.86 & 0.72 & 0.77 \\
\hline
\end{tabular}

Table 5: Error rates (\%) of various normalization methods on CASIA database.

\begin{tabular}{|c|ccc|ccc|}
\hline & \multicolumn{3}{|c|}{ Euclidean } & \multicolumn{3}{c|}{ MQDF } \\
\cline { 2 - 7 } & chn & nccf & grdg & chn & nccf & grd-g \\
\hline LN & 11.38 & 10.46 & 10.31 & 4.11 & 3.49 & 3.54 \\
NLN & 5.44 & 4.48 & 4.90 & 2.26 & 1.75 & 2.00 \\
MN & 5.61 & 4.89 & 4.90 & 2.50 & 2.04 & 2.06 \\
BMN & 5.30 & 4.54 & 4.56 & 2.35 & 1.93 & 1.92 \\
MCBA & 5.48 & 4.73 & 4.83 & 2.31 & 1.87 & 1.96 \\
\hline LDPI & $\mathbf{4 . 4 9}$ & $\mathbf{3 . 6 9}$ & 4.21 & $\mathbf{1 . 9 6}$ & $\mathbf{1 . 5 2}$ & $\mathbf{1 . 7 5}$ \\
2MN & 4.99 & 3.97 & 4.33 & 2.24 & 1.70 & 1.91 \\
2BMN & 4.63 & 3.71 & $\mathbf{4 . 0 7}$ & 2.15 & 1.62 & 1.76 \\
2CBA & 4.75 & 3.95 & 4.25 & 2.11 & 1.68 & 1.78 \\
\hline
\end{tabular}

On fixing the direction resolution (12-direction) and sampling mesh $(7 \times 7)$, we combine the three types of direction features with nine normalization methods. The weight parameter $w_{0}$ of pseudo 2D normalization was set to 0.75 for good recognition performance [24]. The error rates on the test sets of two databases are listed in Table 4 and Table 5, respectively. In the tables, the pseudo $2 \mathrm{D}$ normalization methods P2DMN, P2DBMN, and P2DCBA are denoted by $\mathbf{2} \mathbf{M N}, \mathbf{2 B M N}$, and $\mathbf{2} \mathbf{C B A}$, respectively, for saving space. Comparing the normalization methods, we can see that pseudo 2D methods are superior to $1 \mathrm{D}$ ones, and the linear normalization $(\mathrm{LN})$ is inferior to other $1 \mathrm{D}$ normalization methods. To compare the three types of features, let us view the error rates of $1 \mathrm{D}$ normalization methods and those of pseudo 2D methods separately.

It can be seen in Table 4 and Table 5 that with $1 \mathrm{D}$ normalization, the NCCF and the gradient fea- ture perform comparably, and both outperform the chaincode feature. Four normalization methods, namely, NLN, MN, BMN, and MCBA, perform comparably. With pseudo 2D normalization, the NCCF perform best, and the gradient feature outperforms the chaincode feature. Comparing the pseudo 2D normalization methods, LDPI and P2DBMN outperform P2DMN and P2DCBA (especially on the CASIA database). On both two databases, the best performance is yielded by the NCCF with LDPI normalization, and the NCCF with P2DBMN is competing.

The gradient feature performs comparably with NCCF with 1D normalization but inferiorly with pseudo 2D normalization. This is because pseudo 2D normalization, though equalize the stroke density better than 1D normalization, also deforms the stroke directions remarkably. While the gradient feature describes the deformed stroke directions, NCCF takes the stroke directions of original image.

\subsection{Computation times}

To compare the computational complexity of normalization methods, we profile the processing time in two sub-tasks: coordinate mapping and normalized image generation, and the latter is dichotomized into binary image and gray-scale image. Smoothing is involved in binary normalized image, but not for gray-scale image.

On the test samples of CASIA database, we counted the CPU times on Pentium-4-3GHz processor. The average times per sample are shown in Table 6 . We can see that the processing time of coordinate mapping varies with the normalization method. The linear normalization (LN) is very fast. NLN is more expensive than other 1D methods, and LDPI is more expensive than other pseudo $2 \mathrm{D}$ methods. This is because NLN and LDPI involve line density computation. Nevertheless, all these normalization methods are not computationally expensive. Normalized image generation for pseudo 2D normalization methods is very time consuming because it involves quadrilateral decomposition. The processing time of binary normalized image includes a smoothing time of about $0.3 \mathrm{~ms}$.

The CPU time of feature extraction is almost independent of normalization method. It has two parts: directional decomposition and blurring. The average CPU times of three direction features are shown in Table 7 . The processing time of blurring depends on the sparsity of direction planes (zero pixels are not considered). The direction planes of chaincode feature are sparse, and those of gra- 
Table 6: Average CPU times (ms) of normalization on CASIA database. Binary normalized image involves smoothing.

\begin{tabular}{|c|ccc|}
\hline & coordinate & binary & grayscale \\
\hline LN & 0.002 & 0.318 & 0.133 \\
NLN & 0.115 & 0.331 & 0.143 \\
MN & 0.017 & 0.321 & 0.126 \\
BMN & 0.024 & 0.332 & 0.135 \\
MCBA & 0.032 & 0.336 & 0.137 \\
\hline LDPI & 0.266 & 1.512 & 1.282 \\
P2DMN & 0.143 & 1.514 & 1.236 \\
P2DBMN & 0.147 & 1.536 & 1.274 \\
P2DCBA & 0.156 & 1.542 & 1.261 \\
\hline
\end{tabular}

dient feature are densest. The directional decomposition of NCCF is most time-consuming because it involves line segment decomposition, and gradient direction decomposition is more expensive than chaincode decomposition. Overall, NCCF is still the most computationally efficient because it saves the time of normalized image generation. The average CPU time of NCCF ranges from $1.21 \mathrm{~ms}$ to $1.47 \mathrm{~ms}$, covering coordinate mapping and feature extraction. For reference, the average classification time of MQDF (with cluster-based candidate selection) for 3,755 classes is $3.63 \mathrm{~ms}$, which can be largely reduced by fine implementation, however.

Table 7: Average CPU times (ms) of feature extraction on CASIA database.

\begin{tabular}{|c|cc|}
\hline & direction & blurring \\
\hline chn & 0.121 & 0.439 \\
nccf & 0.458 & 0.752 \\
grd-g & 0.329 & 1.276 \\
\hline
\end{tabular}

\subsection{Performance on unconstrained handwriting}

The error rates of the best methods on handprinted characters, say, $0.64 \%$ on ETL9B database and $1.52 \%$ on CASIA database, are fairly high. To test the performance on unconstrained handwritten characters, we use the classifiers trained with the training set of CASIA database to classify the samples in a small data set, which contains 20 samples for each of 3,755 classes, written by 20 writers without constraint. Some samples of the unconstrained set are shown in Fig. 13.

On the unconstrained set, we evaluate various nor-

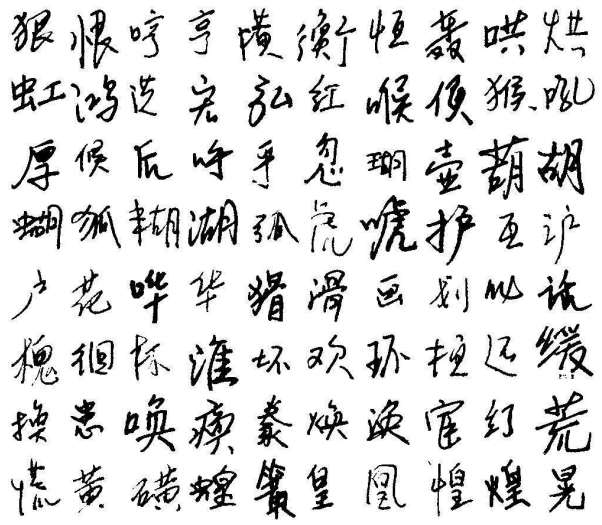

Figure 13: Samples of unconstrained handwritten characters.

malization methods with two good features: NCCF and gradient direction feature. The error rates are listed in Table 8 . We can see that as for handprinted characters, the pseudo $2 \mathrm{D}$ normalization methods yield lower error rates than their $1 \mathrm{D}$ counterparts on unconstrained characters as well. However, line-density-based nonlinear normalization methods, NLN and LDPI, are evidently inferior to the centroid-based methods. The P2DBMN method performs best, and the P2DCBA method is competitive. Comparing the two types of direction features, the gradient features outperforms NCCF with 1D normalization methods, but with pseudo $2 \mathrm{D}$ normalization, NCCF is superior. Overall, the error rates on unconstrained characters are still very high.

Table 8: Error rates (\%) on unconstrained handwritten Chinese characters.

\begin{tabular}{|c|cc|cc|}
\hline & \multicolumn{2}{|c|}{ Euclidean } & \multicolumn{2}{c|}{ MQDF } \\
\cline { 2 - 5 } & nccf & grdg & nccf & grd-g \\
\hline LN & 36.04 & 35.44 & 21.80 & 21.46 \\
NLN & 26.61 & 27.04 & 16.23 & 16.96 \\
MN & 25.63 & 25.18 & 16.40 & 16.09 \\
BMN & 25.15 & 24.53 & 15.91 & 15.64 \\
MCBA & 25.53 & 25.06 & 15.76 & 15.66 \\
\hline LDPI & 24.93 & 25.60 & 15.12 & 15.73 \\
P2DMN & 23.48 & 24.38 & 14.84 & 15.37 \\
P2DBMN & $\mathbf{2 2 . 7 5}$ & $\mathbf{2 3 . 3 5}$ & $\mathbf{1 4 . 2 7}$ & $\mathbf{1 4 . 6 4}$ \\
P2DCBA & 23.80 & 23.78 & 14.73 & 14.79 \\
\hline
\end{tabular}

Fig. 14 shows some misclassified samples by MQDF with the best normalization-feature combination (P2DBMN and NCCF). Most of the misclassified characters are similar in shape to the assigned class: some are inherently similar (top three rows in Fig. 14), and some others are similar due to cursive writing (fourth and fifth rows). Yet the characters 
of the bottom row are not highly similar to their assigned classes.

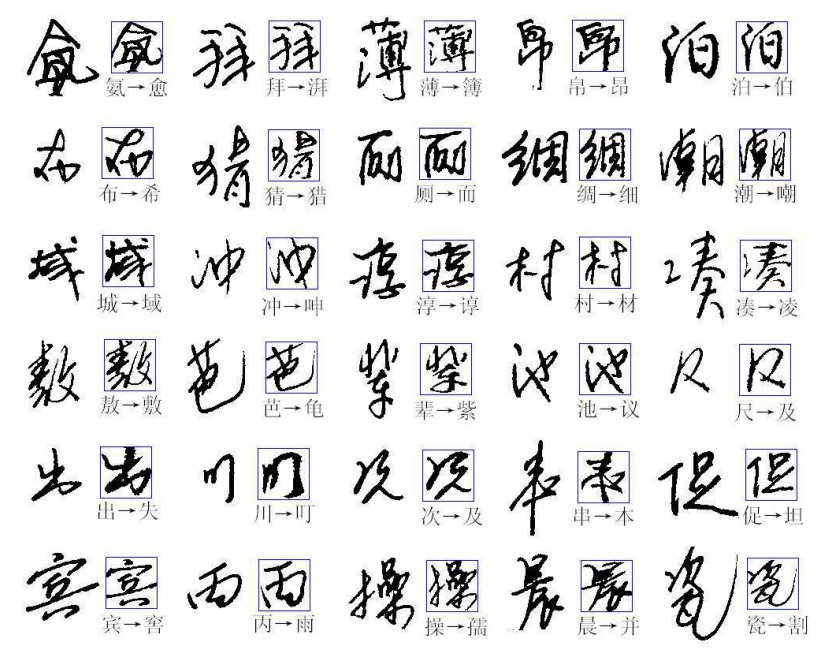

Figure 14: Misclassified samples (each shown original image and normalized image) of unconstrained handwritten characters.

\section{Concluding Remarks}

We compared various shape normalization and selected feature extraction methods in offline handwritten Chinese character recognition. For direction feature extraction, our results show that 12-direction and 16-direction features outperform 8-direction feature, and the 12-direction feature has better tradeoff between accuracy and complexity. The comparison of normalization methods shows that pseudo 2D normalization methods outperform their 1D counterparts. On handprinted characters, a line-densitybased method LDPI and a centroid-based method P2DBMN perform best. Comparing three types of direction features, the NCCF and the gradient feature outperform the chaincode feature, compete when 1D normalization is used, but with pseudo $2 \mathrm{D}$ normalization, the NCCF outperforms the gradient feature. Overall, the best normalization-feature combinations are LDPI and P2DBMN with NCCF.

We also tested the performance on a small set of unconstrained handwritten characters, with classifiers trained with handprinted samples. The error rates on unconstrained characters are very high, but the comparison of normalization and feature extraction methods reveals new insights. Though pseudo 2D normalization methods again outperform their 1D counterparts, the line-density-based LDPI method is inferior to the centroid-based methods, and the P2DBMN method performs best. The best normalization-feature combination on unconstrained characters is P2DBMN with NCCF.

Training classifiers with unconstrained handwritten samples will be able to improve the accuracy of unconstrained character recognition. To collect large database of unconstrained characters is an urgent task in the near future. To reduce the error rate to a fairly low level (say, $2 \%$ on isolated characters), however, simply using the current normalization and feature extraction methods and training current classifiers with larger sample set will not suffice. The methods of shape normalization, feature extraction, and classifier design need to be reconsidered for better recognizing cursively written characters. Training classifiers discriminatively can improve the accuracy of both handprinted and unconstrained character recognition.

\section{Acknowledgements}

This work is supported in part by the Hundred Talents Program of Chinese Academy of Sciences and the Natural Science Foundation of China (grant no.60543004).

\section{References}

[1] R. Casey and G. Nagy, Recognition of printed Chinese Characters, IEEE Trans. Electronic Computers 15(1) (1966) 91-101.

[2] W. Stalling, Approaches to Chinese character recognition, Pattern Recognition 8 (1976) 8798.

[3] S. Mori, K. Yamamoto, and M. Yasuda, Research on machine recognition of handprinted characters, IEEE Trans. Pattern Anal. Mach. Intell. 6(4) (1984) 386-405.

[4] T.H. Hildebrandt and W. Liu, Optical recognition of Chinese characters: advances since 1980, Pattern Recognition 26(2) (1993) 205-225.

[5] M. Umeda, Advances in recognition methods for handwritten Kanji characters, IEICE Trans. Information and Systems E29(5) (1996) 401410 .

[6] C.-L. Liu, S. Jaeger, and M. Nakagawa, Online recognition of Chinese characters: the state-ofthe-art, IEEE Trans. Pattern Anal. Mach. Intell. 26(2) (2004) 198-213. 
[7] K. Fukunaga, Introduction to Statistical Pattern Recognition, 2nd edition (Academic Press, 1990).

[8] R.O. Duda, P.E. Hart, and D.G. Stork, Pattern Classification, 2nd edition (Wiley Interscience, 2001).

[9] A.K. Jain, R.P.W. Duin, and J. Mao, Statistical pattern recognition: a review, IEEE Trans. Pattern Anal. Mach. Intell. 22(1) (2000) 4-37.

[10] I.-J. Kim and J.H. Kim, Statistical character structure modeling and its application to handwritten Chinese character recognition, IEEE Trans. Pattern Anal. Mach. Intell. 25(11) (2003) 1422-1436.

[11] T. Iijima, H. Genchi, and K. Mori, A theoretical study of the pattern identification by matching method, in Proc. First USA-JAPAN Computer Conference, Oct. 1972, 42-48.

[12] M. Yasuda and H. Fujisawa, An improvement of correlation method for character recognition, Trans. IEICE Japan J62-D(3) (1979) 217-224 (in Japanese).

[13] Y. Yamashita, K. Higuchi, Y. Yamada, and Y. Haga, Classification of handprinted Kanji characters by the structured segment matching method, Pattern Recognition Letters 1 (1983) 475-479.

[14] H. Fujisawa and C.-L. Liu, Directional pattern matching for character recognition revisited, in Proc. 'Tth Int'l Conf. on Document Analysis and Recognition, Edinburgh, Scotland, 2003, 794798.

[15] J. Tsukumo and H. Tanaka, Classification of handprinted Chinese characters using nonlinear normalization and correlation methods, in Proc. 9th Int'l Conf. on Pattern Recognition, Rome, 1988, 168-171.

[16] H. Yamada, K. Yamamoto, and T. Saito, A nonlinear normalization method for hanprinted Kanji character recognition - line density equalization, Pattern Recognition 23(9) (1990) 10231029 .

[17] F. Kimura, K. Takashina, S. Tsuruoka, and Y. Miyake, Modified quadratic discriminant functions and the application to Chinese character recognition, IEEE Trans. Pattern Anal. Mach. Intell. 9(1) (1987) 149-153.
[18] F. Kimura, T. Wakabayashi, S. Tsuruoka, and Y. Miyake, Improvement of handwritten Japanese character recognition using weighted direction code histogram, Pattern Recognition 30(8) (1997) 1329-1337.

[19] N. Kato, M. Abe, Y. Nemoto, A handwritten character recognition system using modified Mahalanobis distance, Trans. IEICE Japan J79-D-II(1) (1996) 45-52.

[20] R.G. Casey, Moment normalization of handprinted character, IBM J. Res. Develop. 14 (1970) 548-557.

[21] C.-L. Liu, H. Sako, and H. Fujisawa, Handwritten Chinese character recognition: alternatives to nonlinear normalization, in Proc. 'Th Int'l Conf. on Document Analysis and Recognition, Edinburgh, Scotland, 2003, 524-528.

[22] C.-L. Liu and K. Marukawa, Global shape normalization for handwritten Chinese character recognition: a new method, in Proc. 9th Int'l Workshop on Frontiers of Handwriting Recognition, Tokyo, Japan, 2004, 300-305.

[23] T. Horiuchi, R. Haruki, H. Yamada, and K. Yamamoto, Two-dimensional extension of nonlinear normalization method using line density for character recognition, in Proc. 4th Int'l Conf. on Document Analysis and Recognition, Ulm, Germany, 1997, 511-514.

[24] C.-L. Liu and K. Marukawa, Pseudo twodimensional shape normalization methods for handwritten Chinese character recognition, Pattern Recognition 38(12) (2005) 2242-2255.

[25] M. Hamanaka, K. Yamada, and J. Tsukumo, Normalization-cooperated feature extraction method for handprinted Kanji character recognition, in Proc. 3rd Int'l Workshop on Frontiers of Handwriting Recognition, Buffalo, NY, 1993, 343-348.

[26] C.-L. Liu, K. Nakashima, H. Sako, and H. Fujisawa, Handwritten digit recognition: benchmarking of state-of-the-art techniques, Pattern Recognition 36(10) (2003) 2271-2285.

[27] A. Kawamura, K. Yura, T. Hayama, Y. Hidai, T. Minamikawa, A. Tanaka, and S. Masuda, On-line recognition of freely handwritten Japanese characters using directional feature densities, in Proc. 11th Int'l Conf. on Pattern Recognition, The Hague, 1992, Vol.2, 183186. 
[28] G. Srikantan, S.W. Lam, and S.N. Srihari, Gradient-based contour encoder for character recognition, Pattern Recognition 29(7) (1996) 1147-1160.

[29] C.-L. Liu, K. Nakashima, H. Sako, and H. Fujisawa, Handwritten digit recognition: investigation of normalization and feature extraction techniques, Pattern Recognition 37(2) (2004) 265-279.

[30] N. Hagita, S. Naito, and I. Masuda, Handprinted Chinese characters recognition by peripheral direction contributivity feature, Trans. IEICE Japan J66-D(10) (1983) 1185-1192 (in Japanese).

[31] M. Yasuda, K. Yamamoto, H. Yamada, and T. Saito, An improved correlation method for handprinted Chinese character recognition in a reciprocal feature field, Trans. IEICE Japan J68-D(3) (1985) 353-360.

[32] L.-N. Teow and K.-F. Loe, Robust vision-based features and classification schemes for off-line handwritten digit recognition, Pattern Recognition 35(11) (2002) 2355-2364.

[33] M. Shi, Y. Fujisawa, T. Wakabayashi, and F. Kimura, Handwritten numeral recognition using gradient and curvature of gray scale image, Pattern Recognition 35(10) (2002) 2051-2059.

[34] X. Wang, X. Ding, and C. Liu, Gabor filterbase feature extraction for character recognition, Pattern Recognition 38(3) (2005) 369-379.

[35] C.-L. Liu, M. Koga, and H. Fujisawa, Gabor feature extraction for character recognition: comparison with gradient feature, in Proc. 8th Int'l Conf. on Document Analysis and Recognition, Seoul, Korea, 2005, 121-125.

[36] C.-L. Liu, M. Koga, and H. Sako, H. Fujisawa, Aspect ratio adaptive normalization for handwritten character recognition, in Advances in Multimodal Interfaces-ICMI2000, T. Tan, Y. Shi, W. Gao (Eds.), LNCS Vol. 1948, Springer, 2000, 418-425.

[37] C.-L. Liu, High accuracy handwritten Chinese character recognition using quadratic classifiers with discriminative feature extraction, in Proc. 18th Int'l Conf. on Pattern Recognition, Hong Kong, 2006, Vol.2, 942-945.

[38] H. Liu and X. Ding, Handwritten character recognition using gradient feature and quadratic classifier with multiple discrimination schemes, in Proc. 8th Int'l Conf. on Document Analysis and Recognition, Seoul, Korea, 2005, 19-23.

[39] C.-L. Liu, Y-J. Liu, and R-W. Dai, Preprocessing and statistical/structural feature extraction for handwritten numeral recognition, in Progress of Handwriting Recognition, A.C. Downton and S. Impedovo (Eds.), (World Scientific, 1997) 161-168.

[40] J. Guo, N. Sun, Y. Nemoto, M. Kimura, H. Echigo, and R. Sato, Recognition of handwritten characters using pattern transformation method with cosine function, Trans. IEICE Japan J76-D-II(4) (1993) 835-842 (in Japanese).

[41] T. Wakabayashi, S. Tsuruoka, F. Kimura, and Y. Miyake, On the size and variable transformation of feature vector for handwritten character recognition, Trans. IEICE Japan J76-DII(12) (1993) 2495-2503 (in Japanese).

[42] R.V.D. Heiden, and F.C.A. Gren, The Box-Cox metric for nearest neighbor classification improvement, Pattern Recognition 30(2) (1997) 273-279.

[43] N. Kato, M. Suzuki, S. Omachi, H. Aso, and Y. Nemoto, A handwritten character recognition system using directional element feature and asymmetric Mahalanobis distance, IEEE Trans. Pattern Anal. Mach. Intell. 21(3) (1999) 258-262. 